IZA DP No. 7498

Mit Neo-Korporatismus durch die Krise?

Die Rolle des Sozialen Dialogs in

Deutschland, Österreich und der Schweiz

Werner Eichhorst

J. Timo Weishaupt

July 2013 


\title{
Mit Neo-Korporatismus durch die Krise? Die Rolle des Sozialen Dialogs in Deutschland, Österreich und der Schweiz
}

\author{
Werner Eichhorst \\ IZA
}

\author{
J. Timo Weishaupt \\ Universität Mannheim
}

Discussion Paper No. 7498
July 2013

IZA

P.O. Box 7240

53072 Bonn

Germany

Phone: +49-228-3894-0

Fax: +49-228-3894-180

E-mail: iza@iza.org

Any opinions expressed here are those of the author(s) and not those of IZA. Research published in this series may include views on policy, but the institute itself takes no institutional policy positions. The IZA research network is committed to the IZA Guiding Principles of Research Integrity.

The Institute for the Study of Labor (IZA) in Bonn is a local and virtual international research center and a place of communication between science, politics and business. IZA is an independent nonprofit organization supported by Deutsche Post Foundation. The center is associated with the University of Bonn and offers a stimulating research environment through its international network, workshops and conferences, data service, project support, research visits and doctoral program. IZA engages in (i) original and internationally competitive research in all fields of labor economics, (ii) development of policy concepts, and (iii) dissemination of research results and concepts to the interested public.

IZA Discussion Papers often represent preliminary work and are circulated to encourage discussion. Citation of such a paper should account for its provisional character. A revised version may be available directly from the author. 


\section{ABSTRACT

\author{
Mit Neo-Korporatismus durch die Krise? Die Rolle des \\ Sozialen Dialogs in Deutschland, Österreich und der Schweiz
}

Dieser Artikel untersucht die Rolle der Sozialpartner bei der Bewältigung der Wirtschaftskrise 2008/09 in Deutschland, Österreich und der Schweiz. Die Fallstudien zeigen, dass zu Beginn der Krise eine schnelle Reaktion der Politik auf das gemeinsame Handeln von Arbeitgeberverbänden, Gewerkschaften und Regierungen zurückzuführen ist. Eine zentrale Rolle kam dabei den Sozialpartnern jener Sektoren zu, die am stärksten von der Krise betroffenen waren. In der Schweiz und Österreich wurden vorhandene Verhandlungsstrukturen genutzt, während in Deutschland stärker informelle Kanäle genutzt wurden. Den Sozialpartnern gelang es dabei, ihre in den Jahren vor der Krise entwickelten sektoralen Strukturen wettbewerbsfähiger Produktion mit Hilfe staatlicher Unterstützung über die Krise hinweg zu stabilisieren. Darin drückt sich zunächst eine zeitlich und thematisch begrenzte Interessenkonvergenz von Arbeitgebern, Gewerkschaften und Regierung aus, allerdings mit einem Potential für eine künftige Revitalisierung der Sozialpartnerschaft und neokorporatistischer Konzertierungen.

\section{Crisis Response in Neo-corporatist Countries: A Comparative Analysis of Social Dialogue in Germany, Austria and Switzerland}

This article analyses the role of the social partners in weathering the Great Recession 2008/09 in Germany, Austria and Switzerland. The case studies show an initial phase of largely consensus-based fiscal expansion and adjustment of labour market policies such as short-time work programs. Social partners of those sectors most affected by the crisis played a crucial role. Policy formulation was either based on well-established corporatist interest intermediation procedures (Austria and Switzerland) or on more informal interactions (Germany). With the aid of the state, the social partners were able to stabilize the structures built prior to the crisis. On the one hand, this is an expression of temporarily and thematically converging interests between employers, labour unions and governments. On the other hand, it represents a window of opportunity to revive recently declining social partnership institutions and neocorporatist concertation.

JEL Classification: J52, J53, J58

Keywords: Sozialpartnerschaft, Neokorporatismus, Krise, Wandel, Kontinentaleuropa

Corresponding author:

Werner Eichhorst

IZA

P.O. Box 7240

53072 Bonn

Germany

E-mail: eichhorst@iza.org 


\section{Einleitung}

Seit Beginn der Weltwirtschafts- und Finanzkrise, die 2009 ihren vorläufigen Höhepunkt erfahren hatte, befinden sich nahezu alle westlichen Demokratien in einer prekären Situation: enorme Haushaltsdefizite gehen mit steigender (Langzeit- und Jugend-)Arbeitslosigkeit einher. Während die Südeuropäer besonders unter der Krise leiden - aber auch die nordischen und angelsächsischen Volkswirtschaften von hoher Arbeitslosigkeit betroffen sind - bilden insbesondere die deutschsprachigen Volkswirtschaften eine Ausnahme. Deutschland, welches lange Zeit als Europas „kranker Mann“ beschrieben wurde, entpuppte sich nahezu über Nacht zum Musterschüler mit einem Rekordstand bei der Erwerbstätigkeit und kontinuierlich sinkender Arbeitslosigkeit (Eichhorst 2011). Mit nahezu konstant bleibender Vollbeschäftigung - sogar während des (vorläufigen) Krisenhöhepunkts im Jahre 2009 - sind aber auch Österreich und die Schweiz bemerkenswerte Sonderfälle.

Erklärungen über die Ursachen des deutschen „Beschäftigungswunders“ finden sich bereits in großer Vielzahl (Caliendo/Hogenacker 2012, Möller 2010, Rinne/Zimmermann 2013).

Gemeinsamer Tenor dieser Studien ist, dass nicht nur eine Vielzahl von wirtschaftlichen und politischen Einflüssen in synergetischer Weise zusammenspielten, sondern dass auch und gerade die Kooperation zwischen Politik und Arbeitgeber- und Arbeitnehmerverbänden entscheidend war, um eine arbeitsmarktpolitische Krise abzuwenden. Dass solche „konzertierte Aktionen“ stattfanden ist zunächst überraschend, denn in allen drei Ländern verlieren nicht nur die Verbände selbst seit Jahren an organisatorischer Macht und politischer Legitimation, sondern die Politik bevorzugte politische „Alleingänge“ bei nahezu allen bedeutenden Reformen des Sozialstaats. Während in den 1990er noch eine gewisse Euphorie für sozialpartnerschaftliche Konzertierung in vielen Ländern Europas herrschte, scheint diese seit der ersten Dekade des neuen Jahrtausends „nicht nur in Deutschland wieder abzuebben“ (Jochem 2009: 210). Daher stellt sich die Frage, warum es in diesen drei Ländern zu einer konsensualen Lösung in der Beschäftigungspolitik im Kontext der Weltwirtschaftskrise kam. Nach Beantwortung dieser Frage mit Hilfe neokorporatistischer Theorieansätze soll auch diskutiert werden, ob sich die „Institution“ der tripartistischen Sozialpartnerschaft als im Laufe der Krisenbewältigung (nachhaltig) gewandelt hat (Haipeter 2012; Urban 2010). Wobei sich diese Frage sicherlich (noch) nicht endgültig beantworten lässt, diskutieren wir im Lichte der hier aufgeführten Evidenz 
die Aspekte, die für einen weiteren Abbau, eine grundsätzliche Revitalisierung oder eine temporäre Öffnung sprechen.

Die Evidenz dieses Artikels stützt sich auf eine Kombination mehrerer Quellen, einschließlich einer Analyse von Printmedien ${ }^{1}$, einer Auswertung von Berichten und Stellungnahmen der Sozialpartner, Forschungsinstitute und politischen Einrichtungen, sowie einer kleiner Zahl vertraulich geführter Interviews mit Vertretern aus Politik und Verbänden. ${ }^{2}$

\section{Neokorporatismustheorie und rivalisierende Hypothesen}

Unter dem Begriff des Neokorporatismus verstehen wir die Einbindung von organisierten Interessen, insbesondere der Sozialpartner (Arbeitgeber- und Arbeitnehmerverbände), in die Politikformulierung und ihre Umsetzung. In der Literatur wird zwischen Makro-, Meso-, und Mikrokorporatismus unterschieden. Unser Fokus liegt ausschließlich auf der Makroebene und somit in der Aushandlung von Politik zwischen Staat (Bundesregierung) und Verbänden, welche sowohl als Interessenvertreter ihrer Mitglieder (logic of membership) wie auch als Interessenvermittler (logic of influence) agieren (Andersen/Woyke 2003; Schmitter/Streeck 1999).

Für die deutsche Geschichte schreibt Bernhard Weßels (2000: 7), dass sich die „Konzertierung von Interessen häufig als gutes Instrument erwiesen“ hat, um soziale und wirtschaftliche Herausforderungen zu bewältigen. Die Organisationsdichte und organisationale Stärke der Verbände hat sich dabei in der Nachkriegsgeschichte als institutioneller Wettbewerbsvorteil herausgestellt (Hall/Soskice 2001), da es die Herausbildung eines angebotsorientierten Korporatismus ermöglichte, welcher maßgeblich für den Erfolg der deutschen „diversifizierten Qualitätsproduktion“ verantwortlich war (Streeck 1991). Ähnliche Entwicklungen finden sich auch in Österreich (Heinisch 2001) und der Schweiz (Katzenstein 1987). Während die Sozialpartner bis in die 1980er ihren Einfluss nutzen konnten, um Politik nach ihren Präferenzen mitzugestalten, wurde ihr Mitwirken seit den 1990ern vermehrt zur Disposition gestellt,

\footnotetext{
${ }^{1}$ Für Deutschland wurden insbesondere, aber nicht ausschließlich die Frankfurter Allgemeine Zeitung, das Handelsblatt und die Süddeutsche Zeitung verwendet; für Österreich Der Standard; für die Schweiz die Neue Züricher Zeitung.

${ }^{2}$ Für eine Auflistung der geführten Interviews, s. Anhang.
} 
einerseits, da sie organisational geschwächt und innerlich geteilt auftraten (Thelen/van Wijnbergen 2003), und andererseits, da im Zuge des post-keynesianischen Wirtschaftsverständnis unter dem Primat der Preisstabilität ihre Rolle an Legitimation verlor. Im deutschen Kontext ist insbesondere das Scheitern der Konzertierung unter der Kohl-Regierung im Jahre 1996 und des Bündnisses für Arbeit der Regierung Schröder 2002/3 Ausdruck eines politischen Einflussverlustes der Sozialpartner (Streeck/Hassel 2003). Auch die große Koalition hat diesen Eindruck nur verstärkt, denn der sozialdemokratische Arbeitsminister Franz Müntefering führte die „Rente mit 67“ nicht nur gegen den Willen der Gewerkschaften ein, sondern informierte diese nicht einmal über seine Pläne (Streeck 2009:62).

Nicht nur die deutsche Politik der jüngeren Vergangenheit zeichnet sich durch staatliche „Alleingänge“ aus (Weishaupt 2010; 2011a), auch die Rentenreformen aus den Jahren 2000, 2003 und 2004 in Österreich folgten diesem Muster (Busemeyer 2005; Obinger/Tálos 2006). Folglich wurde auch in Österreich eine „Abkehr“, „Erosion“, „Zerbröseln“ oder zumindest das „Wanken“ des Neo-Korporatismus festgestellt (Armingeon 2005; Engel 2006; Streeck 2005; Tálos/Fink 2003). Aber auch in der Schweiz gibt es Anzeichen für die Abschwächung korporatistischer Regierungsformen. Einerseits werden die Sozialpartner, insbesondere die Gewerkschaftsbewegung, in der öffentlichen Wahrnehmung zunehmend durch „,neue soziale Bewegungen“ ersetzt (Armingeon 2001:406). Andererseits beklagen die Politik und die Öffentlichkeit den korporatistischen „Filz“ welcher zu politischer Trägheit und Ineffizienz führt (Armingeon 2003:311).

Aber auch die „bipartistische“ Sozialpartnerschaft steht vor praktischen und strategischen Herausforderungen. Einerseits verlieren die Verbände durch den anhaltenden Mitgliederschwund in Gewerkschaften und Austritten von Unternehmen aus den Arbeitgeberverbänden an organisationaler Verhandlungsmacht ${ }^{3}$ (Silvia 2010; Streeck 2009; Visser 2009). Andererseits bilden sich im Kontext der globalen Arbeitsteilung, hoher Kapitalmobilität und des Primats der „permanenten Austerität“ (Pierson 2001) neue Produzentenkoalitionen aus Arbeitgebern und Gewerkschaften bzw. Betriebsräten, sowohl auf sektoraler als auch auf betrieblicher Ebene, welche wiederum neue Anpassungsstrategien hervorbrachten. Diese Strategien beinhalten oft die

\footnotetext{
${ }^{3}$ In Österreich besteht nach wie vor eine Pflichtmitgliedschaft für Unternehmen in der Wirtschaftskammer Österreich (WKÖ).
} 
Inkaufnahme von Segmentierung von Arbeitsmärkten und eine Dualisierung von Belegschaften in Insider und Outsider (Eichhorst/Marx 2010; Palier/Thelen 2010). Wenn Sozialpartnerschaft dann zu einer Zunahme der Outsider führt, wird diese potentiell als Repräsentation von Partikularinteressen wahrgenommen und kann an gesamtgesellschaftlicher Akzeptanz verlieren. Durch die vermehrte Bildung von (streikwilligen) Sparten- oder Berufsgewerkschaften wird diese Wahrnehmung noch zusätzlich verstärkt (Dörre 2011). Insgesamt stehen die Sozialpartner, insbesondere die Gewerkschaften, aufgrund dieser politischen, organisatorischen und normativen Trends unter Druck, und die Institution der Sozialpartnerschaft befindet sich tendenziell in einem Prozess der „exhaustion“ (Streeck/Thelen 2005) bzw. Erschöpfung, welcher - über einen längeren Zeitraum hinweg - zur Selbstauflösung der Institution der Sozialpartnerschaft führen könnte (Streeck 2009; Trampusch 2009).

Warum aber sollten sich Regierungen überhaupt auf eine tripartistische Konzertierung - gerade in Krisenzeiten - mit den Sozialpartnern einlassen? Hierzu finden sich in der NeokorporatismusForschung mehrere Erklärungsansätze. Zu den bekanntesten gehört sicherlich die These des „politischen Tauschhandel“ (Lehmbruch 1979; Pizzorno 1978; Schmitter/Lehmbruch 1979). Schmitter, Pizzorno und Lehmbruch argumentieren, dass ein politischer Tauschhandel in erster Linie von Regierungen angestrebt wird, um sozialen und industriellen Frieden zu sichern, während die Sozialpartner sich Mitbestimmungsmöglichkeiten erkaufen. Hier wird explizit angenommen, dass ein Tauschgeschäft auch Kompromisse beinhaltet und somit Gewinne und Verluste für alle Seiten entstehen. Der neokorporatistische Tauschhandel der 1970er ähnelt somit den „sozialen Pakten“ der 1990er. Im Kontext von sozialen Pakten werden oftmals moderate Tarifabschlüsse von Gewerkschaften akzeptiert, da im Gegenzug sozialstaatliche Konzessionen von Seiten der Regierung angeboten und von Arbeitgebern mitgetragen werden (Avdagic 2010). Daher werden soziale Pakte auch als Form eines „,kompetitiven Korporatismus“ verstanden (Rhodes 2000).Die nationale Wettbewerbsfähigkeit wird durch eine Reduktion der Lohnstückkosten durch sozialstaatlichen Ausbau (oder zumindest eine Minimierung an Kürzungen) von den Gewerkschaften „erkauft“ (Rhodes 2000). Auch kann eine Politik im Interesse der Produzentenkoalitionen zu erheblichen Belastungen für die öffentlichen oder sozialstaatlichen Haushalte führen, wie es das Beispiel der Frühverrentung zeigte (Ebbinghaus 2006). 
Wie Afonso (2013) darstellt, sind diese „funktionalistischen“ bzw. „machtpolitischen“ Thesen nicht die einzige Erklärung des Neokorporatismus. Soziale Konzertierung findet unter Umständen auch aus informationspolitischen oder Legitimation stiftenden Überlegungen heraus statt. Genauer gesagt sieht Pepper Culpepper (Culpepper 2002: 775) eine tripartistische Konzertierung als kognitive Ressource, wobei Bernhard Ebbinghaus (2010: 256) die Schaffung von „shared understandings“ betont. Informationen und Deliberation schaffen also eine breitere Datengrundlage und ein besseres Verstehen von Problemen und möglichen Antworten, was wiederum Kompromisse entstehen lässt und Lösungsansätze tragfähiger macht. Letztlich ist noch eine wahltaktische Erklärung seitens der Politik denkbar. Im Gegensatz zum Tauschhandel (welcher aus einer „Schwäche“ der Regierung gesucht wird) und dem kognitiven Korporatismus (welcher aus pragmatischen Überlegungen gewählt wird), sucht die Regierung die Einbindung der Sozialpartner lediglich um politisch kontroverse Reformen an die Öffentlichkeit verkaufen zu können („,blame avoidance“ durch möglichst breiten Konsens) (Hassel 2009: 10). Durch die exit option der Regierungen sehen sich in diesem Fall die Sozialpartner gezwungen zu kooperieren, da eine Verweigerung der Teilnahme eine Einflussnahme ganz ausschließen würde.

Insgesamt kann also festgehalten werden, dass Hypothesen zu neokorporatistischen Konzertierungen sich im Hinblick auf die Motivation und Macht der Akteure beziehen. Nur wenn starke Akteure sich Gewinne versprechen (z.B. Einfluss auf Politikgestaltung, Wählergunst etc.) oder wenn schwache Akteure die Nicht-Teilnahme als noch größeren Verlust wahrnehmen, kommt es zu tripartistischer Kooperation.

\section{Unterschiede und Gemeinsamkeiten in Sozialpartnerschaft und Neokorporatismus in Deutschland, Österreich und der Schweiz}

Der Begriff der Sozialpartnerschaft wird sowohl in Deutschland als auch Österreich und der Schweiz gebraucht, auch wenn die Intensität zwischen den Ländern variiert. Mit Sicherheit ist Österreich das Land mit den am meisten ausgeprägten neo-korporatistischen Zügen (Siaroff 1999:184). Einerseits ist der Österreichische Gewerkschaftsbund (ÖGB) stark zentralisiert und sowohl administrativ als auch durch seine Finanz- und Personalhoheit und das Recht zur Bewilligung von Streiks institutionell sehr einflussreich (Klenk et al. 2012:26). Obwohl kein „offizieller“ Sozialpartner, ist auch die institutionelle Mitwirkung der pflichtmitgliedschaftlich 
organisierten Arbeitnehmerkammer (AK) von großer Bedeutung bei der Politikgestaltung, da sie dem ÖGB sehr nahe steht und zusätzliche Ressourcen mobilisiert. Andererseits besteht für alle Unternehmen in Österreich eine Pflichtmitgliedschaft in der Wirtschaftskammer Österreich (WKÖ), wodurch die Arbeitgeber gezwungen sind, gemeinsame Positionen heraus zu arbeiten. Wie die AK für den ÖGB steht die Industriellen Vereinigung (IV), die freiwillige Interessenvertretung der österreichischen Industrie, der WKÖ als starker, inoffizieller Sozialpartner zur Seite und ist sowohl personell wie auch institutionell stark in alle politische Entscheidungsprozesse eingebunden. Der Deckungsgrad von Tariflöhnen, wichtiger Ausdruck für die Stärke der industriellen Beziehungen eines Landes, liegt in Österreich bei nahezu 100 Prozent, während die Gewerkschaftsdichte bei knapp unter 30 Prozent liegt (Visser 2009). Die Sozialpartnerschaft in Österreich zeichnet sich zudem durch eine starke (informelle) Organisation und eine „Kultur“ des Korporatismus aus, welche in einer Vielzahl an bi- und triparitätischen Institutionen zur politischen Entscheidungsfindung zum Ausdruck kommt und auch zu einer engen personelle Verflechtung zwischen Politik und Verbänden führt.

In Deutschland sind die Verbände weitaus schwächer als in Österreich. Der Deutsche Gewerkschaftsbund (DGB) ist weniger zentral organisiert und verfügt über geringere Einflussmöglichkeiten. Im Gegensatz zu Österreich ist zudem die sektorale Segmentierung in Deutschland deutlich stärker erkennbar - und hier die Führungsrolle der Metallindustrie. Zudem ist in den letzten Jahren eine Fragmentierungsbewegung hin zu Spartengewerkschaften bzw. Berufsgewerkschaften erkennbar. Der Deckungsgrad der Tariflöhne liegt bei knapp über 60 Prozent und die Gewerkschaftsdichte bei annähernd 20 Prozent (Visser 2009). Auch auf Seiten der Arbeitgeber finden sich deutliche Unterschiede zu Österreich. Einerseits gibt es in Deutschland keine Pflichtmitgliedschaft in der Bundesvereinigung der Deutschen Arbeitgeberverbände (BDA), was - insbesondere seit der Wiedervereinigung - zu Austritten aus den Arbeitgeberverbänden ${ }^{4}$ und zur Gründung von sogenannten OT-Verbänden „ohne Tarifbindung“ geführt hat. ${ }^{5}$ Andererseits, sind die deutschen Arbeitgeberverbände traditionell dezentraler organisiert, und konfligierende Interessen zwischen großen und kleinen Unternehmen

\footnotetext{
${ }^{4}$ Eine Pflichtmitgliedschaft besteht nach wie vor bei den Industrie- und Handelskammern, welche insbesondere eine Schlüsselrolle bei der Ausgestaltung der dualen Ausbildung spielen und damit Produktions- und Ausbildungsstandards definieren (Schroeder and Weßels 2010, 9).

${ }^{5}$ Obwohl es keine offiziellen Statistiken der Verbände gibt, zeigen empirischen Studien, dass der Mitgliederschwund insbesondere in der Metallindustrie zu finden ist, wohingegen die Mitgliederzahl in der Chemiebranche einen Anstieg verzeichnet (Silvia 2010, 175-8).
} 
sowie zwischen der BDA und dem Bundesverband der Deutschen Industrie (BDI) wurden oft auch in der Öffentlichkeit ausgetragen (Schroeder 2010:38). Trotz der schwächeren Positionierung der deutschen Sozialpartner spielen sie eine wichtige Rolle in der deutschen Politik. Die Sozialpartner sind nicht nur offizielle Mitglieder in den Selbstverwaltungsorganen der deutschen Sozialversicherung - wie dies auch der Fall in Österreich und der Schweiz ist sondern auch die Geschäftsordnungen des Bundestages und der Bundesregierung sehen ausdrücklich ihre Mitwirkung in der Politik vor, was dazu führt, dass die Sozialpartner in der Vorbereitung von Gesetzen durch Stellungnahmen und Anhörungen miteinbezogen werden.

Obwohl auch die Schweiz der Gruppe der koordinierten Marktwirtschaften, oder CMEs, zugeteilt wird (Hall/Gingerich 2009) unterscheidet sich die Schweiz mit Blick auf die für die Sozialpartnerschaft relevanten Politiken von Deutschland und Österreich in vielerlei Hinsicht: Nicht nur ist das Arbeitsrecht deutlich „arbeitgeberfreundlicher“, auch die Lohnverhandlungen zeichnen sich, trotz der Existenz von sektoralen Kollektivverträgen durch eine starke Stellung der betrieblichen Ebene aus (Bender et al. 2007: 172). Zudem gibt es in der Schweiz, im Gegensatz zu Deutschland und Österreich, keinen mitbestimmenden Aufsichtsrat in großen Unternehmen. Der Deckungsgrad der Tariflöhne liegt mit 50 Prozent unterhalb des deutschen Wertes, wobei die Gewerkschaftsdichte bei knapp über 20 Prozent mit Deutschland vergleichbar ist. Hinzu kommt eine starke innergewerkschaftliche Konkurrenz durch eine Zweiteilung der Spitzenverbände in den Schweizer Gewerkschaftsbund (SGB) und Travail.Suisse, die wiederum relativ dezentral organisiert sind und nur wenig Kontrolle über ihre Mitgliedergewerkschaften ausüben können (s. auch Afonso 2013: 30). Die Arbeitgeber hingegen sind in einem kohäsiven und starkem Arbeitgeberverband (SAV) organisiert, der sich seit Mitte der 1990er für eine starke Liberalisierung in der Arbeitsmarktpolitik einsetzt (Oesch 2011: 82). Trotz insgesamt „liberalerer“ institutioneller Ausgestaltung bleibt die Schweiz ein sozialpartnerschaftliches Land aufgrund seiner ausgeprägten Tradition der Konsensbildung und der formalen und informellen Einbindung der Sozialpartner in die Politikgestaltung (Oesch 2007).

Insgesamt bilden die drei deutschsprachigen Länder ein „Kontinuum“ des Neo-Korporatismus mit deutlichen Unterschieden im Hinblick auf Zentralisierung der Verbände, die Institutionalisierung der Politikmitgestaltung und die dominante Verhandlungsebene (Katzenstein 1987). Gemeinsam haben die drei Länder jedoch den günstigen Verlauf der Beschäftigung und 
Arbeitslosigkeit trotz empfindlicher Rückgänge des BIP über die Krisenjahre hinweg, wie Tabelle 1 zeigt. Gleichzeitig ergaben sich aufgrund der Lohnabschlüsse 2008 und niedriger Inflation in Österreich und der Schweiz erhebliche Reallohnsteigerungen 2009, die einerseits die Konsumnachfrage stabilisierten, aber auch - bei geringerer Kapazitätsauslastung - zu einem Anstieg der Lohnstückkosten führten. Diese war auch in Deutschland stark ausgeprägt, da die Arbeitsproduktivität stark einbrach (Tabelle 1).

\section{[Tabelle 1 hier einfügen]}

\section{Deutschland}

Das deutsche Wirtschafts- und Sozialsystem befindet sich seit geraumer Zeit in einem Prozess des Umbaus (Schmidt 2012), welcher auch das Verhältnis zwischen Staat und Verbänden nachhaltig prägte. Insbesondere die zu Beginn der zweiten Amtszeit der rot-grünen Bundesregierung eingeführten Hartz-Reformen (2002-2005) im Kontext der Agenda 2010 wurden zwar von den Arbeitgeber begrüßt, aber die Gewerkschaften fühlten sich trotz anfänglicher Unterstützung zunehmend von den Sozialdemokraten hintergangen. Vor allem der Ausbau flexibler Beschäftigungsverhältnisse, die Hartz-IV-Gesetzgebung und auch das politisch gewollte Erstarken der Finanzmarktakteure (Stichwort Riester-Rente, Lockerung der Finanzmarktregeln und Bankenaufsicht) bei gleichzeitigen Einschnitten in den Sozialversicherungsstrukturen wurde von den Gewerkschaften als direkten Angriff auf den deutschen Sozialstaat gesehen (Weishaupt 2011a). Insgesamt stellte sich große Frustration unter den Gewerkschaften ein, die „privilegierte Partnerschaft“ zwischen Sozialdemokraten und Gewerkschaften war aufgekündigt worden (Urban 2010, 4), und der Neokorporatismus erschien „erschöpft“ (Streeck 2009; Trampusch 2009).

Auch unter den Sozialpartnern selbst fanden große Veränderungen statt. Auf Branchenebene gab es während der ersten Dekade des neuen Jahrtausends einen allgemeinen Trend zur Lohnmoderation, zu einer sinkendenden Reichweite der Tarifverträge und zur Dezentralisierung der Lohnverhandlungen (Streeck 2009). Gerade der letzte Punkt führte dann aber dazu, dass auf der betrieblichen Ebene sozialpartnerschaftliche Gestaltungsmöglichkeiten relevanter wurden, und schon vor der Krise konnte durch Arbeitszeit- und Lohnflexibilisierung Beschäftigung 
gesichert, die Wettbewerbsfähigkeit exportorientierter Unternehmen gesteigert und dank starker Auslastung der Kapazitäten die Arbeitszeitkonten der Belegschaften aufgefüllt werden (Reisenbichler/Morgan 2012).

Insgesamt zeichnete sich folgendes Bild zu Beginn der Krise ab: Aufgrund der guten wirtschaftlichen Entwicklung (Wachstumsraten von über drei Prozent in den Jahren 2006 und 2007) und einem allgemeinen Exportboom, schrieben die meisten (exportorientierten) Unternehmen schwarze Zahlen und waren in einer gefestigten Position. Vor der Krise war nicht länger das früher als zu starr kritisierte Tarifgefüge das Hauptproblem der deutschen Arbeitgeber, sondern der sich abzeichnenden Fachkräftemangel im Kontext einer graduell alternden Bevölkerung. Auf der politischen Ebene bemühten sich Arbeitgeber um weitere Reformen, während die Gewerkschaften vor allem das Ziel der „Schadensbegrenzung“ verfolgten, d.h. den Reformeifer der Regierung zu stoppen oder gar umzukehren (z.B. Rente mit 67), die langfristige Lohnzurückhaltung zu überwinden und neue Felder zu erschließen, in welchen gewerkschaftsrelevante Sozialpolitik ausgebaut (z.B. gesetzlicher Mindestlohn) und neue Themen medienwirksam diskutiert werden können (z.B. Altersarmut, Ausweitung prekärer Arbeitsverhältnisse).

Als die Krise im Sommer 2008 in vielen europäischen Staaten bereits zu einem Anstieg der Arbeitslosigkeit führte, schien Deutschland zunächst verschont zu bleiben. Im Spätherbst setzte dann aber auch in Deutschland eine gewisse Nervosität ein als zahlreiche Firmen ihre Gewinnprognosen nach unten korrigierten, die Produktion drosselten, Zeitarbeiter entließen und Kurzarbeit beantragten oder „Zwangsurlaub“ durch Werksferien verordneten. Im Oktober erwog Arbeitsminister Olaf Scholz (SPD) dann einen „Schutzschirm für Beschäftigung“, welcher insbesondere die Erhöhung der Bezugsdauer der Kurzarbeit von 12 auf bis zu 24 Monate beinhaltete (Berliner Zeitung vom 29.10.2008). Nach internen Beratungen verabschiedete die Bundesregierung dann am 5. November 2008 ein im internationalen Vergleich moderates Konjunkturpaket, welches vor allem über steuerliche Maßnahmen (Entlastungen für Unternehmen und private Haushalte, direkte Investitionshilfen, befristete Kfz-Steuerbefreiung) den Konsum stabilisieren sollte. Daneben wurde aber auch die Bezugsdauer des Kurzarbeitergeldes von 12 auf zunächst 18 Monate verlängert und die Weiterqualifizierung während der Kurzarbeit gefördert. Kurzarbeit, ein Instrument das sich schon in früheren Krisen bewährt hatte, wurde von den Arbeitgeberverbänden, vornehmlich der Metall- und 
Elektrobranche (Gesamtmetall), sowie der Gewerkschaften, insbesondere IG Metall und IG BCE begrüßt. Während die Arbeitgeber ihre qualifizierten Arbeitskräfte durch das Konjunkturtief halten wollten (und damit insbesondere an staatlicher Beteiligung an den Kosten interessiert waren) (Möller 2010), lag das Hauptinteresse der Gewerkschaften in einem Vermeiden oder wenigstens Hinauszögern von Entlassungen.

Da die meisten Beteiligten über das „Tempo des konjunkturellen Abschwungs überrascht“ waren (FAZ, 1.11.2008, S. 12), wurden noch im November insbesondere gewerkschaftliche Stimmen laut, die ein weiteres Konjunkturpaket forderten. Der IG Metall-Vorsitzende Berthold Huber wagte Anfang Dezember einen Vorstoß, indem er ein 7-Punkte-Programm zur Sicherung von Arbeitsplätzen präsentierte, in welchem er sich unter anderem für die Anwendung von arbeitsmarktpolitischen und tarifvertraglichen Instrumenten zur Vermeidung von betrieblichen Kündigungen aussprach und auch eine „Umweltprämie“ vorschlug, welche die Verschrottung von Alt-Autos und den Kauf von Neuwagen anregen sollte (IG Metall Pressemitteilung Nr. 66, 11.12.2008). Bereits einen Tag später forderte dann auch der DGB eine „Verschrottungsprämie“ sowie ein weiteres, größeres Konjunkturpaket in Höhe von 60 Milliarden Euro (DGBPressemitteilung vom 12.12.2008). Dass gerade die Automobilbranche durch ein spezielles Instrument gefördert werden sollte, wurde durch ihre Systemrelevanz begründet - sie sei „Rückgrat der Wirtschaft“. Die Prämie fand laute Unterstützung von Matthias Wissmann, CDUMitglied und Präsident des Deutschen Automobilverbands (VDA).

Obwohl Bundeskanzlerin Angela Merkel (CDU) und Bundesfinanzminister Peer Steinbrück (SPD) zunächst jegliche Diskussion um ein weiteres Konjunkturpaket bis Januar 2009 kategorisch ausschlossen (Süddeutsche, 28.11.2008, S. 5), suchte die Bundesregierung das Gespräch mit den Vertretern der Wirtschaft und den Verbänden im Kanzleramt. Auf einem bis in die Abendstunden andauerten „Konjunkturgipfel“ berieten - und verhandelten - die Kanzlerin, Vizekanzler Steinmeier, die wichtigsten Ressort-Chefs, die Vorstandsvorsitzende einiger DAX30 Unternehmen (z.B. Siemens, Telekom), den größten Banken, sowie die Spitzen aller großen Arbeitgeber- und Gewerkschaftsverbände über Möglichkeiten zur Vermeidung betriebsbedingter Kündigungen. Auf diesem Treffen kündigte die Industrie eine freiwillige Selbstverpflichtung an, keine Massenentlassungen vorzunehmen (Süddeutsche, 16.12.2008, S. 5). Diese Selbstverpflichtung war allerdings gekoppelt an weitere Konzessionen beim Kurzarbeitergeld 
und Fortbildungsmaßnahmen, was damit bereits die Weichen für ein 2. Konjunkturpaket in Aussicht stellte. Zudem waren Kündigungen im Notfall zulässig („Katastrophenklausel“) und auch Kündigungen für Zeitarbeiter nicht ausgeschlossen (FAZ, 17.12.2008, S. 4). Am darauffolgenden Freitag lud dann Bundesarbeitsminister Olaf Scholz (SPD) die Personalvorstände der DAX-30 Unternehmen nach Berlin ein, um weitere Details zu besprechen und nach weiteren Möglichkeiten zur Stabilisierung der Beschäftigung zu suchen (Süddeutsche, 20.12.2008, S. 23). In dem Treffen versicherte Scholz, dass Unternehmen die Arbeitsplätze sichern, besonders von der Kurzarbeit profitieren könnten, während aber Handwerkspräsident Otto Kentzler Versprechungen über „Jobgarantien“ ausschloss (Hamburger Abendblatt, 22.12.2008).

Am 12.1.2009 beschloss die Bundesregierung dann ein zweites Konjunkturprogramm, welches u.a. öffentliche Investitionen in Bildung, Infrastruktur und Informationstechnologie und eine Verbesserung der Kreditversorgung von Unternehmen umfasste, aber auch eine von den Sozialpartner gemeinsam geforderte Neugestaltung der Kurzarbeit (Verfahrensvereinfachung und eine Übernahme von 50 Prozent der Sozialversicherungsbeiträge seitens der BA), eine Erhöhung der Mittel für Qualifizierungsmaßnahmen der Bundesagentur für Arbeit, und die befristete Einführung einer PKW-Umweltprämie. Damit realisierten insbesondere die Metall-, Elektro- und Chemiebranchen ihre Präferenzen, also die Branchen, die auch im Wesentlichen das Instrument der Kurzarbeit nutzten. Dass die PKW-Umweltprämie realisiert wurde ist nicht zuletzt auf IG Metall Vorstand Berthold Huber zurückzuführen (Die Zeit Online, 2.7.2009).

Die rasche Einigung auf ein zweites Konjunkturpaket und dessen reibungslose Umsetzung ist somit insbesondere der konsensualen Zusammenarbeit der Sozialpartner und der pragmatischen und konstruktiven Rolle der Gewerkschaften zu verdanken. Während in anderen Ländern Gewerkschaften mit Streiks drohten, unterstützten die deutschen Gewerkschaften nicht nur offiziell die arbeitsmarktpolitischen Maßnahmen der Bundesregierung, sondern akzeptierten auch auf Branchen- und Betriebsebene Lohneinbußen um Arbeitsplätze zu sichern (Interview mit Huber, Die Zeit vom 30.4.2009). Durch diese enge und konstruktive Zusammenarbeit wurde auf Bundesebene ein informelles „Bündnis für Arbeit“ ausgearbeitet und politische Tauschgeschäfte ausgehandelt, und damit letztlich auch der Raum geschaffen, weitere Bündnisse auf Branchenund Betriebsebene in den Jahren 2009 und 2010 zu realisieren. Auf Bundesebene kam es zwar 
nochmals Ende April 2009 zu einem Krisengipfel mit etwa 40 Teilnehmern. Doch es sprachen sich nicht nur die Vertreter der Wirtschaft und die Arbeitgeberverbände gegen ein drittes Konjunkturpaket aus, auch die Kanzlerin, Finanzminister Steinbrück und Wirtschaftsminister zu Guttenberg waren sich einig, dass die Instrumente zunächst wirken müssten, bevor über neue Maßnahmen beschlossen werden könne (Berliner Zeitung, 23. April 2009). Das Treffen war zumindest zum Teil von der Kanzlerin strategisch angesetzt worden, um die gewerkschaftlichen Forderungen noch weiteren Milliarden-Investitionen sowie die Rufe nach Steuersenkungen und Unternehmenssteuerreform der Arbeitgeber zu unterminieren. Einig waren sich die Sozialpartner aber darin, dass die Bezugsdauer der Kurzarbeit von 18 bis auf 24 Monate verlängert werden sollte, und dass Unternehmen (auch die, die Kurzarbeiter nicht weiterqualifizieren) die Sozialabgaben nach sechs Monaten Kurzarbeit komplett erstattet bekommen sollten. Da sowohl Scholz bereits im Februar einen solchen Schritt favorisierte und auch Wirtschaftsminister zu Guttenberg die Idee „sympathisch“ fand, folgte dann Ende Mai die Umsetzung per Rechtsverordnung (Spiegel Online, 24.9.2009).

Der deutsche Arbeitsmarkt erholte sich dann auch rasch im zweiten Halbjahr 2009. Insgesamt konnten durch die sozialpartnerschaftlichen und gesetzgeberischen Lösungsansätze über eine halbe Million Arbeitsplätze im Krisenjahr 2009 gesichert werden. Die Wirkung der Abwrackprämie wird auf zwischen 20.000 (Autoanalyst Jürgen Pieper) und 200.000 (VizeKanzler Steinmeier und IG Metall-Chef Huber) Arbeitsplätze geschätzt (Der Spiegel Online, 15.08.2009), während die Kurzarbeit zusammen mit anderen Maßnahmen zum Erhalt von Beschäftigung (Arbeitszeitkonten und Überstundenabbau) etwa 600.000 Arbeitsplätze sicherte (Möller 2010).

Trotz der relativen positiven Entwicklung auf dem Arbeitsmarkt fielen die Regierungsparteien CDU/CSU und SPD in ein historisches Tief bei den Bundestagswahlen im September, während die Freien Demokraten (FDP) mit knapp 15 Prozent ein Rekordergebnis erzielten. Aufgrund der veränderten Machtverhältnisse im Parlament kam es dann zur Bildung einer „schwarz-gelben“ Regierungskoalition zwischen CDU/CSU und FDP. Trotz neuer Regierungskonstellation gab es eine gewisse Kontinuität, denn auch die neue Regierung verzichtete nicht darauf, Sonderregelungen für die Kurzarbeit zu gewähren: das Kabinett beschloss bereits im November die Bezugsdauer auf 18 Monate für Anträge im Jahre 2010 festzulegen (nicht aber die 
Kostenerstattung der Sozialabgaben). Zudem lud die Kanzlerin Anfang Dezember 2009 die Vertreter der Wirtschaft und Verbände erneut zu einem „Wirtschafts- und Kreditgipfel“ ins Kanzleramt ein. Im Gegensatz zum Konjunkturgipfel vom Dezember 2008, war dieser vier Stunden andauernde Gipfel allerdings weniger als Diskussions-, sondern eher als Informationsforum und Presse-Event gedacht und ein weiteres Konjunkturpaket war bereits im Vorfeld kategorisch ausgeschlossen worden (FAZ 3.12.2009). Der Gipfel diente somit insbesondere wahltaktischen Zwecken - die Kanzlerin startet gemeinsam in das neue Jahr bekundete aber auch öffentlich den Willen der schwarz-gelben-Regierung die Sozialpartner nach wie vor in das politische Geschehen auf höchster Ebene einzubinden.

Im Ergebnis sah das im Anschluss in Kraft tretende Wachstumsbeschleunigungsgesetz vom 1.1.2010 zwar keinerlei Förderungen für die Industrie mehr vor (mit Ausnahme von Steuerbegünstigungen für Hoteliers). Im Sommer 2010 allerdings wurden dann die Sonderregelungen für die Kurzarbeit nochmals um 15 Monate bis März 2012 verlängert, was auf Druck der Arbeitgeberverbände, insbesondere seitens der BDA und Metaller, sowie der Gewerkschaften geschah. Während Teile der Union und die FDP der Kurzarbeit zunehmend kritisch gegenüberstanden, fürchteten sie dass die Metall- und Elektroindustrie ihre Beschäftigungspakte aufkündigen könnte wenn die Kurzarbeit nicht länger politisch gefördertwerden würde (Süddeutsche, 20.2.2010). Die zentrale Rolle der Verbände zeigt sich auch in einem Statement eines Interviewpartners: „wenn beide Sozialpartner etwas fordern, dann kann die Politik kaum anders handeln.“ (Interview, DE-1).

Insgesamt spricht also vieles für ein Ende der konsensualen tripartistische Konzertierung nach Antritt der neuen Regierung: die Kanzlerin nutzt Gipfel speziell aus einer wahltaktischen Räson, während die Akteure der Metall- und Elektroindustrie Privilegien durchsetzen, auf Kosten der Regierung und gegen den Willen anderer Branchen. Dennoch gibt es auch Anzeichen dafür, dass die ausgehandelte Krisenreaktion nicht folgenlos für die tripartistische Politikgestaltung und die Sozialpartnerschaft war. Erstens, lässt sich ein allgemeines Hervorheben der Sozialpartnerschaft als Wettbewerbsvorteil und zentraler Aspekt der Krisenbewältigung konstatieren, nicht nur von den Sozialpartnern selbst, sondern auch von führenden Persönlichkeiten innerhalb der beiden großen Volksparteien. Damit zeichnet sich zweitens ein neues, zumindest rhetorisches, Paradigma für zukünftige Krisen ab, welches die Sozialpartner in die Pflicht nimmt, 
verantwortungsvolle und konsensuale Lösungen zu finden. Gleichzeitig werden aber auch klare Erwartungshaltungen für wirtschaftlich gute Zeiten gesetzt, denn es gilt gerade in den guten Zeiten in Sozialpartnerschaft zu investieren, um in Zeiten der Krise belastbare Partnerschaften zu haben (Rede von Bundeskanzlerin Angela Merkel anlässlich der 100. Internationalen Arbeitskonferenz der Internationalen Arbeitsorganisation). ${ }^{6}$ Damit ist ein starkes, normatives Fundament geschaffen worden, welches eine Abkehr der Sozialpartnerschaft und der Konzertierung zunehmend erschwert, während ein Wiedererstarken der Tarifpartner nach einer langen Erosionsphase erstmals wieder möglich erscheint. Obwohl es noch zu früh ist, die Auswirkungen der Krise mit Sicherheit abzuschätzen, wird bereits deutlich, dass die bei der Krisenüberwindung maßgeblich involvierten Gewerkschaften IG BCE und IG Metall seit 2009 ihren Mitgliederschwund überwunden haben und neue Mitglieder rekrutieren konnten (FAZ vom 15.9.2011, S.13; Handelsblatt vom 4.12.2012 (online)). Generell zeigten sich Gewerkschaften während der Krise in einem Umfragehoch: Im März 2009 hatten 39 Prozent der Bundesbürger „großes“ oder „sehr großes“ Vertrauen in die Gewerkschaften (Infratest dimap). ${ }^{7}$ Eine zumindest zeitweise - Intensivierung, nicht Ab- oder Umkehr der Sozialpartnerschaft ist damit die Folge der Krise.

\section{5 Österreich und Schweiz im Vergleich mit der Bundesrepublik Deutschland}

\section{1 Österreich}

In Österreich war die Zeit der Koalition von Christdemokraten (ÖVP) und den rechtspopulistischen Freiheitlichen (FPÖ/BZÖ) bis Anfang 2007 von einer gewissen Zurückdrängung der Sozialpartner geprägt, insbesondere des gewerkschaftlichen Einflusses, auf die Gestaltung von gesetzlichen Regelungen in der Sozialpolitik (Obinger/Tálos 2006). Die Schwächung der Gewerkschaften auf politischer Ebene wurde zudem noch durch den hausgemachten „BAWAGSkandal“" verstärkt, in welchen der ÖGB verwickelt war (Engel 2006, Interviews AT-1 und AT2). Die Sozialpartnerschaft in anderen Bereichen, auch in der Tarifpolitik, zeichnete sich hingegen durch eine bemerkenswerte Kontinuität aus, gerade in den industriellen Kernbereichen. Mit dem Regierungswechsel hin zu einer erneuten großen Koalition von Sozialdemokraten (SPÖ)

\footnotetext{
${ }^{6}$ Abrufbar auf: http://www.bundesregierung.de/Content/DE/Rede/2011/06/2011-06-14-merkelilo.html;jsessionid=B68C19ECF617676617D6750AC48AB3BE.s2t2. Download am 19.12.2012.

${ }^{7}$ Vergleiche: http://www.infratest-dimap.de/service/presse/aktuell/vertrauen-der-buerger-in-die-politik-gestiegen/. Download am 19.12.2012.
} 
und ÖVP wurde die Rolle der beiden Sozialpartner wieder aufgewertet und stabilisiert. Gleichzeitig konnten in der Arbeitsmarkt- und Sozialpolitik auch vor 2007 einige Dinge konstruktiv und kooperativ geregelt werden, so die Reform des Kündigungsschutzes („Abfertigung neu“), die Einbeziehung der freien Dienstnehmer in die Sozialversicherung, die Ausweitung der Arbeitslosenversicherung für Selbstständige oder neue Regelungen zur Zeitarbeit sowie die Einführung eines generellen kollektivvertraglichen Mindestlohnes.

Die Krisenreaktion in Österreich gründete sich, ähnlich wie in Deutschland, auf ein Zusammenspiel von mehreren Konjunkturpaketen zur Stabilisierung der Wirtschaft einerseits und spezifischen Maßnahmen für den Arbeitsmarkt andererseits. Österreich profitierte dabei zu Beginn der Krise von einem ersten Maßnahmenpaket, das kurz vor vorgezogenen Neuwahlen im September 2008, noch vor dem akuten wirtschaftlichen Einbruch, im Parlament beschlossen worden war (Anhebung der Pensionen, Familienbeihilfe). Gleichzeitig wirkten zu Beginn der Krise die im Vorjahr angesichts eines günstigen Konjunkturverlaufs vereinbarten Lohnerhöhungen noch nach (Interview AT-3). Ähnlich wie in Deutschland reagierte die Politik rasch mit Konjunkturpaketen auf die krisenhafte Entwicklung der Wirtschaft und die befürchtete Verschlechterung der Lage auf dem Arbeitsmarkt (Der Standard, 3.7.2009; 30.7.2009; 28.8.2009). In Österreich sind hier vor allem die beiden von Regierung und Parlament Konjunkturpakete I und II vom Oktober 2008 bzw. Februar 2009 sowie das zusätzliche Arbeitsmarktpaket vom Juni 2009 zu nennen (vgl. auch BMWFJ 2009). Diese umfassten neben Infrastrukturprojekten und Investitionsanreizen u.a. auch arbeitsmarktpolitische Initiativen wie die Erleichterung und Ausweitung der Kurzarbeiterregelung, der sogenannten Bildungskarenz, der Arbeitsstiftungen und anderer arbeitsmarktpolitischer Programme. Hinzu kam eine von 2010 auf 2009 vorgezogene Steuerreform, die eine Entlastung der Arbeitnehmerhaushalte umfasste (Breuss et al. 2009). Während bereits die Regierung von SPÖ und ÖVP unter dem damaligen Bundeskanzler Alfred Gusenbauer (SPÖ) wieder eine stärkere Beteiligung der Sozialpartner an der Politikformulierung angestrebt hatte, verstärkte sich diese Entwicklung mit der erneuten großen Koalition unter Bundeskanzler Werner Faymann (SPÖ), die ab Anfang Dezember 2008 im Amt war (Interviews AT-1 und AT-2). Während der Wirtschaftsminister der ÖVP Reinhold Mitterlehner aus der Leitung der Wirtschaftskammer in die Regierung wechselte, wurde der frühere Vorsitzende des ÖGB Rudolf Hundstorfer Arbeits- und Sozialminister. Insgesamt drückte sich darin die starke informelle Koordinierung der Wirtschaftspolitik über personelle und 
institutionelle Verflechtungen zwischen Regierung und Sozialpartnern aus. In der frühen, fiskalpolitisch expansiven Phase zu Beginn der Krise ergab sich rasch ein „,keynesianischer Konsens“ zwischen allen maßgeblichen Akteuren, also Arbeitgeber- und Arbeitnehmerseite sowie den beiden regierenden Parteien.

Wie in Deutschland lag eine Ausweitung der Kurzarbeit im gemeinsamen Interesse der Sozialpartner, insbesondere der exportorientierten Metall- und Elektroindustrie, um Fachkräfte über die Krise im Betrieb halten zu können (Der Standard, 10.7.2009). Dieser gemeinsamen Position folgend wurden die Bedingungen für Kurzarbeit in Österreich im ersten Halbjahr 2009 im Zuge der Konjunkturpakete verändert. Dies umfasste die Verlängerung der Bezugsdauer von 12 auf zunächst 18 und später 24 Monate, die Ergänzung um berufliche Qualifizierung und die Übernahme der Arbeitgeberbeiträge ab dem 7. Monat der Kurzarbeit. Auch wurden für 2009 und 2010 zusätzliche Mittel für die Kurzarbeit bereitgestellt (Der Standard 3.7.2009). Die dennoch begrenzte Rolle der Kurzarbeit mit einem maximalen Bestand von etwa 56.000 gemeldeten und 37.000 realisierten Kurzarbeitsfällen ${ }^{8}$ Mitte 2009, kann mit der insgesamt kleineren Rolle von industriellen Großbetrieben in Österreich einerseits und der institutionellen Ausgestaltung der Kurzarbeit und anderen Formen der flexiblen Anpassung der Unternehmen an konjunkturelle Schwankungen andererseits erklärt werden (Bock-Schappelwein et al. 2011; Der Standard, 29.12.2009). ${ }^{9}$ Im Vergleich zu Deutschland war das Instrument der Kurzarbeit in Österreich weniger in der betrieblichen Praxis etabliert, und die Kosten der Kurzarbeit blieben trotz der Reformen insgesamt für Arbeitgeber höher als in Deutschland (Der Standard, 26.6.2009). Weiterhin hing die Nutzung der Kurzarbeit von einer kollektivvertraglichen Regelung ab, die durch eine Vereinbarung auf betrieblicher Ebene konkretisiert wird. Schließlich setzt die Kurzarbeit in Österreich eine Nachbeschäftigungsfrist nach Ende der Kurzarbeit voraus (Interviews AT-1 und AT-3).

Neben der Kurzarbeit ist in Österreich die „Bildungskarenz“ als staatliches Instrument zur zeitlich befristeten Freistellung von Personal in Verbindung mit Weiterbildung zu nennen. Ähnlich wie bei der Kurzarbeit wurden die Bedingungen für die Gewährung einer Freistellung

\footnotetext{
${ }^{8}$ Das entspricht 1,2\% der unselbstständig Beschäftigen ohne Beamte in Österreich; im Kontrast zu 3,9\% der sozialversicherungspflichtig Beschäftigten in Deutschland (Bock-Schappelwein et al. 2011).

${ }^{9}$ Dem entspricht auch die im Frühjahr 2009 eingeführt österreichische Variante einer Abwrack- bzw. Ökoprämie, die auf 1.500 EUR pro Altwagen und 30.000 Fälle beschränkt war. Sie wurde je zur Hälfte vom Staat bzw. vom Autohandel finanziert.
} 
für Weiterbildung im Zuge der Krise Anfang 2009 erleichtert. Beispielsweise wurde die Beschäftigungszeit im Unternehmen von einem Jahr auf sechs Monate verkürzt, die notwendig ist, um einen Anspruch auf den Bildungsurlaub entstehen zu lassen. Die Mindestdauer der Urlaubsphase wurde von drei auf zwei Monate herabgesetzt. Somit konnte eine größere, wenngleich immer noch überschaubare Anzahl von Beschäftigten die Bildungskarenz wahrnehmen, 2009/10 maximal 6.000 bis 7.000 Personen (Der Standard, 29.12.2009). Ab 2008 stand das Weiterbildungsgeld in Höhe der Arbeitslosenunterstützung für alle Nutzer der Bildungskarenz aus Mitteln der Arbeitsmarktpolitik zur Verfügung, während die Kosten der Qualifizierung aus Mitteln der Länder, teilweise auch von den Arbeitgebern getragen wurden, die ebenfalls ein gewisses Interesse an der verstärkten Nutzung der Bildungskarenz hatten, um den Arbeitsausfall abzufedern. Was die vermittelten Bildungsinhalte angeht, so nimmt die österreichische Bildungskarenz wenige Festlegungen vor. Es müssen lediglich mindestens 20 Stunden in der Woche für Weiterbildung verschiedenster Art schriftlich nachgewiesen werden, es besteht jedoch keine Anforderung an den Erwerb oder die Vertiefung betrieblich relevanter Qualifikationen. Damit kann die Bildungskarenz in gewissem Rahmen vor allem als eine von Arbeitgeber- und Arbeitnehmerseite gemeinsam getragene Form zeitlich befristeter Beurlaubung angesehen werden (Interviews AT-1 und AT-2).

Während Kurzarbeit und Bildungskarenz dazu dienten, fortbestehende Arbeitsverhältnisse mit reduzierten Arbeitszeiten bzw. zeitweiser Freistellung zu stabilisieren, konnten die von der Krise in Österreich betroffenen Unternehmen auch dank des im Vergleich zu Deutschland weniger strikten Kündigungsschutzes leichter eine Anpassung über die Belegschaftsgröße vornehmen. Hier sind neben dem starken Gewicht saisonaler Beschäftigung die so genannten „Aussetzverträge“ zu nennen (Interview AT-2, Der Standard, 26.6.2009). Dabei handelt es sich um Entlassungen in den Bezug von Arbeitslosengeld in Verbindung mit einer Wiedereinstellungszusage, die in der Regel nach drei Monaten wirksam wird. Allerdings werden die kurzzeitig arbeitslos gemeldeten Personen nicht vom österreichischen Arbeitsmarktservice, wie sonst üblich, aktiviert und in andere Stellen vermittelt, sondern sie stehen der Wiedereinstellung durch ihren früheren Arbeitgeber zur Verfügung. Bei der Entlassung werden jedoch alle Ansprüche auf Resturlaub, Sonderzahlungen oder Abfertigung abgerechnet. 2008 und 2009 nahm die Zahl der Arbeitslosen mit Wiedereinstellungszusage deutlich zu, insbesondere im Bereich der vom Konjunktureinbruch am stärksten betroffenen Metallindustrie, ohne dass es 
hierbei zu gesetzlichen Änderungen gekommen wäre. Es gab zumindest eine informelle Verständigung mit den Betriebsräten und der sozialpartnerschaftlich gesteuerten öffentlichen Arbeitsverwaltung (Arbeitsmarktservice, AMS) (Der Standard 26.6.2009). Die Aussetzverträge lagen primär im Interesse der Arbeitgeber, die damit angesichts nicht ausgelasteter Kapazitäten ihre Arbeitskosten - zu Lasten der Arbeitslosenversicherung - kurzfristig reduzieren konnten, ohne ihre im Betrieb eingearbeitetes Fachpersonal dauerhaft zu verlieren. Dies gelang nur, da die sonst üblichen Prinzipien der frühen Aktivierung suspendiert wurden. Die Wirtschaftskammer warb auf verschiedenen Ebenen für die Aussetzverträge und gab Hinweise zur rechtlich korrekten Ausgestaltung.

Im Bereich der Arbeitsmarktpolitik hat Österreich mit der „Ausbildungsgarantie“ ein Programm implementiert, um einerseits die traditionell niedrige Jugendarbeitslosigkeit weiterhin gering zu halten und andererseits dem Fachkräftemangel vorzubeugen. Die Ausbildungsgarantie bedeutet die Übernahme von nicht in eine betriebliche Ausbildung vermittelten Jugendlichen in überbetriebliche Lehreinrichtungen, die dann auch zu einem anerkannten Ausbildungsabschluss führen, ggf. mit Vorbereitungskursen. Die Ausbildungsgarantie wurde zwar bereits vor der Krise eingeführt, hat aber 2008 und 2009 im Zuge der Konjunkturpakete an Bedeutung gewonnen (Der Standard, 2.4.2009; 12.9.2009).

Nach der kurzen fiskalpolitisch expansiven Phase zu Beginn der Krise traten auch in Österreich wieder Bemühungen um die Konsolidierung der öffentlichen Haushalte in den Vordergrund. Damit löste sich auch der fiskal- und arbeitsmarktpolitisch expansive „Krisenkonsens“ wieder auf, und die erwartbaren Interessengegensätze zwischen den Parteien, aber auch zwischen den Sozialpartnern prägten die Debatte wieder stärker. Während die Gewerkschaften gegen Kürzungen zulasten der Arbeitnehmer und Transferbezieher mobilisierten und sich für eine stärkere Belastung höherer Einkommen und Vermögen stark machten, setzten sich bei den tatsächlichen Entscheidungen über den Haushalt eher die Positionen der Arbeitgeberseite durch (Interview AT-3). Dies drückte sich etwa in Einschnitten bei den Sozialleistungen und steuerlichen Begünstigungen für Arbeitnehmer aus. Die letzten Jahre waren damit von den „normalen“ Interessenkonflikten und Aushandlungsprozessen gekennzeichnet. Dabei spielt aber die Sozialpartnerschaft nach wie vor eine zentrale Rolle in Österreich, dies kommt auch in 
gemeinsamen Positionen, etwa Ende 2011 zur Reform der Invaliditätspensionen, zum Ausdruck (Interviews AT-1 und AT-2, Der Standard, 11.2.2012).

\subsection{Schweiz}

Was die Schweiz angeht, so ist zunächst festzuhalten, dass der wirtschaftliche Einbruch Ende 2008 und 2009 insgesamt wesentlich milder verlaufen ist als in Deutschland und Österreich, wie die vorliegenden Daten zeigen. Während die exportorientierte schweizerische Chemie- und Pharmaindustrie aufgrund ihrer geringen Preissensibilität kaum betroffen war und die Binnenund die Bauwirtschaft sich dank der massiven Zuwanderung recht stabil entwickelten, betraf der Nachfrageeinbruch auch in der Schweiz vor allem die Metall- und Elektroindustrie, die über Zulieferbeziehungen mit Deutschland eng verbunden ist (Interview CH-1). Der Konjunktureinbruch traf auf eine Situation der Sozialpartnerschaft, die sich nach einer stärker krisenhaften Entwicklung in den 1990er Jahre wieder stabilisiert hatte, auch dank einer Neuausrichtung der Gewerkschaften, welche vermehrt auf gesamtgesellschaftlich relevante Themen und die Mobilisierung im Hinblick auf Referenden setzten (Oesch 2007). $\mathrm{Zu}$ Beginn der Krise trafen die starke und einheitlich agierenden Arbeitgeber- und Industrieverbände (economiesuisse, Arbeitgeberverband, Gewerbeverband) und die beiden Dachverbände der Gewerkschaften (SGB, travail suisse) mit ihrem Anliegen einer konjunkturellen Stabilisierung zunächst auf eine relativ zurückhaltende Position der stets aus den vier wichtigsten Parteien zusammengesetzten Regierung und insbesondere des Staatssekretariats für Wirtschaft (SECO), denn die Regierung und SECO teilten anfänglich noch eine günstigere Einschätzung der weiteren Entwicklung als die Sozialpartner (Interview CH-2). Nachdem aber Anzeichen einer sich verschlechternden Lage auch in der Schweiz deutlich wurden, wurde Ende 2008 auf gemeinsame Anregung von Arbeitgeberverbänden und vor allem der Gewerkschaften mit Unterstützung des Bundesrates und des Staatssekretariats hin ein erstes Stabilisierungspaket und im Sommer 2009 ein zweites Paket zügig verabschiedet, jeweils im Zuge eines verkürzten parlamentarischen Verfahrens als Nachtragshaushalt. Dabei waren vor allem die Gewerkschaften mit Vorschlägen aktiv, während die Arbeitgeberseite tendenziell etwas zurückhaltender agierte (Interview CH-1). Die Einbindung der Sozialpartner in der Bewertung der Problemlage und der Erörterung der notwendigen Maßnahmen war dabei durch regelmäßige Treffen in der (ständigen) außerparlamentarischen „Kommission für Wirtschaftspolitik“ bereits institutionalisiert. In dieser 
Kommission, welche beim SECO und dem Volkswirtschaftsdepartement angesiedelt ist, finden sich sowohl Vertreter der Arbeitgeber, der Gewerkschaften, der Wissenschaft, der Kantone als auch der Bundesverwaltung (Interview $\mathrm{CH}-3$ ).

Die erste Stufe der Stabilisierungsmaßnahmen sah vor allem vermehrte öffentliche Aufträge und Investitionen sowie die Freigabe von Reserven für die Arbeitsbeschaffung vor und war für die generell stark auf Haushaltskonsolidierung ausgerichtete Fiskalpolitik der Schweiz bemerkenswert expansiv. Dies entsprach den Erwartungen der Gewerkschaften, jedoch war auch die Wirtschaftsministerin Doris Leuthard (Christlichdemokratische Volkspartei, CVP) entsprechend positioniert. Auch aus Gründen der politischen Balance zur Stabilisierung der angeschlagenen Großbank UBS wurden vom Bundesrat auch Maßnahmen zur Förderung der Binnennachfrage ergriffen (Interviews CH-2 und CH-3). Weiterhin verzichteten Bund und Kantone in einer abgestimmten Aktion auf eine kurzfristige Budgetkonsolidierung in der Krise, was von Beobachtern auf die Initiative der Gewerkschaften zurückgeführt wird.

Bei der Stabilisierung der Beschäftigung vertraute man ansonsten zunächst auf die einsetzenden Wirkungen der Konjunkturpolitik im In- und Ausland sowie die eingebauten automatischen Stabilisatoren der Arbeitslosenversicherung und der Kurzarbeit und beobachtete die insgesamt recht robuste Entwicklung. Mit dem noch weitgehend konsensuellen zweiten Stabilisierungspaket kam es auch zu einer Ausweitung des bereits bestehenden Instruments der Kurzarbeit, was die maximale Bezugsdauer anging, die in mehreren Schritten auf zunächst 18 und dann auf maximal 24 Monate verlängert wurde (vgl. auch SECO 2012, NZZ, 8.2.2010). Aus Sicht der Arbeitgeber wurde hiermit ein Instrument forciert, was der Sicherung von Fachkräften während einer Phase der Unsicherheit, aufgrund des externen Schocks aber wohl nur zeitlich begrenzten Krise dienen sollte. Aus Sicht der Gewerkschaften hatte die Vermeidung von Arbeitslosigkeit hohe Priorität. Insgesamt konnte durch den Einsatz der Kurzarbeit v.a. in der Metallindustrie nach Schätzungen des SECO etwa ein halber Prozentpunkt Arbeitslosenquote vermieden werden (NZZ, 9.11.2010). Die konjunkturpolitischen Maßnahmen der ersten beiden Pakete und auch die Ausweitung der Kurzarbeit war zwischen Arbeitgebern und Gewerkschaften sowie der Regierung unstrittig, auch im Parlament gab jeweils große Unterstützung für diese Entscheidungen, wenngleich von linken Parteien tendenziell noch weiter gehende Vorschläge gemacht wurden, die sich jedoch gegenüber 
den bürgerlichen Parteien und der Wirtschaft nicht durchsetzen konnten (vgl. z.B. Economiesuisse in NZZ, 10.3.2009).

Generell spielten jedoch arbeitsmarktpolitische Initiativen in der Schweiz nur eine sehr marginale Rolle. Außer der Kurzarbeit wurden im dritten Stabilisierungspaket im Herbst 2009 einige kleinere arbeitsmarktpolitische Maßnahmen angestrebt, jedoch konnte angesichts der relativ günstigen Entwicklung der Beschäftigung hierfür keine klare Unterstützung mehr im Parlament erreicht werden (NZZ, 25.9.2009). Einige kleinere Programme für Jugendliche wurden zeitlich befristet eingerichtet bzw. ausgeweitet, andere Maßnahmen, etwa für Langzeitarbeitslose, jedoch gar nicht umgesetzt (Interview CH-1 und CH-3). Der Grund hierfür lag darin, dass die Konjunktur rascher als erwartet wieder anzog und die Arbeitslosenquote den vom Parlament nach einem Vermittlungsverfahren zwischen beiden Kammern (Nationalrat und Ständerat) festgelegten (und von den Ökonomen erwarteten) Grenzwert von 5\% gar nicht erreichte, der als Voraussetzung für die Nutzung einiger Instrumente vorgesehen war. Hier hatten die Gewerkschaften und ihnen nahestehenden Parteien für ein stärkeres Eingreifen plädiert, waren jedoch am Widerstand der bürgerlichen Parteien gescheitert. Die Problemlage entschärfte sich jedoch mit der günstigen Wirtschaftsentwicklung zusehends. Damit wurde auch klar, dass die von Regierung, Arbeitgebern und Gewerkschaften gemeinsam getragene und primär sektoral ausgerichtete Stabilisierungsstrategie beendet werden konnte.

\section{Diskussion und Zusammenfassung}

Unsere Analyse für Deutschland, Österreich und die Schweiz zeigt deutlich, dass zu Beginn der Krise Ende 2008 in allen drei Ländern eine starke Interessenkonvergenz zwischen der Regierung, den Arbeitgeberverbänden und den Gewerkschaften bestand, die zu einer raschen konjunkturpolitischen Intervention führte. Eine zentrale Rolle spielten in allen drei Ländern die organisatorisch mächtigen Sozialpartner der am stärksten vom Wirtschaftseinbruch betroffenen Metall- und Elektroindustrie. Sie konnten gemeinsam mit den jeweiligen nationalen Regierungen eine Ausweitung des vorhandenen Instruments der Kurzarbeit erreichen, was aus Sicht der Gewerkschaften zur Sicherung von Einkommen und Arbeitsplätzen, aus Sicht der Arbeitgeber zur Stabilisierung qualifizierter und eingearbeiteter Kernbelegschaften das am besten geeignete Mittel darstellte. Aus Sicht der Regierungen wiederum verhinderten Mehraufwendungen für die 
Kurzarbeit aus Mitteln der Arbeitslosenversicherung und steuerfinanzierte Programme wie „Abwrackprämien“ einen Anstieg der Arbeitslosigkeit. Die stark sektorale Einflusslogik der Metall- und Elektroindustrie folgte damit auch aus den gemeinsamen Bemühungen um eine Flexibilisierung und Modernisierung der sektoralen Tarifverträge und betrieblicher Anpassungskanäle hinsichtlich Löhnen und Arbeitszeitmodellen, die insgesamt in den Jahren vor der Krise zu einer Stabilisierung der Sozialpartnerschaft in der Industrie und einem Rückgewinn an internationaler Wettbewerbsfähigkeit beigetragen hatten. Dass die Sozialpartner erfolgreich die Inhalte der Konjunkturpakte beeinflussten steht klar im Gegensatz mit anderen Autoren, die sich mit der Thematik befasst hatten. Waltraut Schelke argumentiert etwa fälschlicherweise, dass „German official responses largely followed the logic of electoral politics mediated by party coalitions....Contrary to expectations in the Varieties of Capitalism literature, corporatist venues played no discernible role in Germany,” (Schelke, 2012:152). ${ }^{10}$

Die Motivation neokorpartistischer Konzertierung lag für alle drei Parteien in der Durchsetzung ihrer jeweiligen Präferenzen. Klare Unterschiede zeigen sich allerdings in den Kanälen der Konzertierung. In Österreich und der Schweiz wurden die etablierten Strukturen der dreiseitigen Beratung und Abstimmung zwischen den Spitzenverbänden der Wirtschaft, der Gewerkschaften und der Regierung genutzt. Während die österreichische Regierung, insbesondere nach den Wahlen im Herbst 2008, auf personelle Verflechtungen in den Spitzen der Ministerien setzten, nutzen die Politikgestalter in der Schweiz die bereits etablierte Kommission für Wirtschaftspolitik zur Klärung beschäftigungspolitischer Fragen. In Österreich und der Schweiz stand somit nicht der Tauschhandel, sondern vielmehr das Schaffen von „shared understandings“ im Vordergrund, wobei allerdings auch kleinere Zugeständnisse gemacht wurden. In Deutschland wurden nicht nur ad hoc informelle Kanäle stärker genutzt, sondern auch gemeinsame Treffen medienwirksam auf höchster Ebene im Kanzleramt organisiert. Wenig überraschend ist daher, dass gerade im deutschen Fall großzügigere Angebote an die Sozialpartner gemacht und auch von diesen genutzt wurden (Kurzarbeit, Abwrackprämie). Im Krisengipfel 2008 kam es zu einem klaren „Tauschhandel“. Durch die Androhung die Beschäftigungspakte aufzukündigen, zeichnet

\footnotetext{
${ }^{10}$ Ähnlich auch Jonas Pontusson und Damian Raess (2012: 15): “we argue that shifts in the interests and power of economic actors must be taken into account to explain broad changes in patterns of crisis response. In particular, we emphasize the political influence of sectorally based coalitions of firms and unionized workers demanding protection or compensation in the 1970s and the absence or weakness of such coalitions in 2008-2010.”
} 
sich der deutsche Fall dann auch von einer gewissen Machtasymmetrie aus, welche die Sozialpartner für eine gewisse Zeit strategisch nutzen konnten, um weitere Zugeständnisse zu sichern. Seit 2011 fielen weitere arbeitsmarkt- und sozialpolitische Reaktionen vergleichsweise marginal aus - auch aufgrund der jeweils stufenweisen Anpassung an die Krise, die dann wiederum überraschend frühzeitig durch eine erneute Wachstumsphase abgelöst wurde.

Damit bleibt die Wirkung der Sozialpartner auf ein breiteres Spektrum an Politikfeldern, etwa im Sinne eines erneuten großen sozialen Paktes, zwar aus. Generell hat die Krise aufgrund ihres abrupten und tiefgreifenden Charakters jedoch gerade am Anfang zu einer gewissen Stärkung oder Wiederbelebung korporatistischer Abstimmungsprozesse geführt. Damit war auch in allen drei Ländern eine begrenzte Aufwertung der Rolle der Gewerkschaften auf politischer Ebene verbunden, die in allen drei Ländern davor aus unterschiedlichen Gründen an Einfluss verloren hatten. Die langfristigen Wirkungen der Krise auf die Sozialpartner sind weniger eindeutig, treten doch die erwartbaren Interessengegensätze gerade bei Konsolidierungspolitik wieder deutlicher zutage, wenngleich weniger massiv als in den südeuropäischen Ländern. Gleichwohl erscheint

die Sozialpartnerschaft als eine wichtige Voraussetzung für die Abfederung der Wirtschaftskrise in den am stärksten betroffenen Sektoren ohne schwerwiegende Folgen für die Arbeitsmärkte, was wiederum den gegenwärtigen fiskalischen und institutionellen Anpassungsdruck vermindert hat. Damit hat die Abstimmung zwischen den Sozialpartnern und den Regierungen auch ihre Belastbarkeit in einer krisenhaften Situation bewiesen.

\section{Literaturverzeichnis}

Afonso, Alexandre (2013): Social Concertation in Times of Austerity. European Integration and the Politics of Labour Market Reforms in Austria and Switzerland. Amsterdam: Amsterdam University Press.

Andersen, Uwe ; Woyke, Wichard (2003): Handwörterbuch des politischen Systems der Bundesrepublik Deutschland. Opladen: Leske+Budrich.

Armingeon, Klaus (2001): „Das Zusammenspiel von langer demokratischer Tradition, direkter Demokratie, Föderalismus und Korporatismus“, in: Rütters, Peter (Hg.) Verbände und Verbandssysteme in Westeuropa. Opladen: Leske + Budrich, 405-426. 
Armingeon, Klaus (2003): „Konzertierung in der Schweiz“, in: Jochem, Sven \& Siegel, Nico A. (Hg.) Konzertierung, Verhandlungsdemokratie und Reformpolitik im Wohlfahrtsstaat: Das Modell Deutschland im Vergleich. Opladen: Leske + Budrich, 311-327.

Armingeon, Klaus (2005): „Probleme des Übergangs vom Korporatismus zum Pluralismus“, in: Ferdinand Karlhofer; Emmerich Tálos (Hg.): Sozialpartnerschaft. Österreichische und Europäische Perspektiven. Hamburg: LIT-Verlag, 135-158.

Avdagic, S. (2010): „When are concerted reforms feasible? Explaining the emergence of social pacts in Western Europe“, Comparative political studies 43: 628-657.

Bender, Christiane; Grassl, Hans; Schaal, Markus (2007): „Der Schweizer Arbeitsmarkt:

Sonderfall unter Modernisierungsdruck“, in: Thomas S. Eberle; Kurt Imhof (Hg.):

Sonderfall Schweiz. Zürich: Seismo Verlag, 172-187.

BMWFJ (2009): Österreichisches Reformprogramm für Wachstum und Beschäftigung 2009.

Bock-Schappelwein, Julia; Mahringer, Helmut; Rückert, Eva (2011): Kurzarbeit in Deutschland und Österreich. Wien: Österreichisches Institut für Wirtschaftsforschung.

Breuss, Fritz; Kaniovski, Serguei; Schratzenstaller, Margit (2009): Gesamtwirtschaftliche Auswirkungen der Konjunkturpakete I und II und der Steuerreform 2009. Juni 2009. Wien: Österreichisches Institut für Wirtschaftsforschung.

Busemeyer, Marius R. (2005): „Pension Reform in Germany and Austria: System Change vs.

Quantitative Retrenchment”, West European Politics 28: 569-591.

Caliendo, Marco ; Hogenacker, Jens (2012): „The German labor market after the Great Recession: successful reforms and future challenges“, IZA Journal of European Labor Studies, 1: 1-24.

Culpepper, Pepper (2002): „Puzzling, Powering, and 'Pacting': The Informational Logic of Negotiated Reforms“, Journal of European Public Policy 9: 774-790.

Dörre, Klaus (2011): „Funktionswandel der Gewerkschaften. Von der intermediären zur fraktalen Organisation“, in: Thomas Haipeter; Klaus Dörre (Hg.): Gewerkschaftliche Modernisierung. Wiesbaden: VS Verlag, 267-301.

Ebbinghaus, Bernhard (2010): „Reforming Bismarckian Corporatism: The Changing Role of Social Partnership in Continental Europe“, in: Palier, Bruno (Hg.): A Long Goodbye to Bismarck? The Politics of Welfare Reform in Continental Europe. Amsterdam: Amsterdam University Press, 255-278. 
Eichhorst, Werner (2011): Vom kranken Mann zum Vorbild Europas: Kann Deutschlands Arbeitsmarkt noch vom Ausland lernen? IZA Standpunkte 46. Bonn: IZA.

Eichhorst, Werner; Marx, Paul (2010) „Whatever Works: Dualization and the Service Economy in Bismarckian Welfare States“, in: Patrick Emmenegger; Silja Häusermann; Bruno Palier; Martin Seeleib-Kaiser (Hg.): The Age of Dualization. Oxford u.a.: Oxford University Press, 73-99.

Engel, Reinhard (2006): Der wankende Riese. Gewerkschaften zwischen Globalisierung und Krise der Sozialpartnerschaft. Wien: Molden Verlag.

Fajertag, Giuseppe; Pochet, Philippe (Hg.) (1997). Social pacts in Europe. Brussels: European Trade Union Institute.

Haipeter, Thomas (2012): „Sozialpartnerschaft in und nach der Krise: Entwicklungen und Perspektiven“, Industrielle Beziehungen 19: 387-411.

Hall, Peter A. ; Soskice, David W. (2001): „An Introduction to Varieties of Capitalism“, in: Hall, Peter A. \& Soskice, David W. (Hg.) Varieties of Capitalism: The Institutional Foundations of Comparative Advantage. Oxford; New York: Oxford University Press, 168.

Hall, Peter A.; Gingerich, Daniel W. (2009): „Varieties of Capitalism and Institutional Complementarities in the Political Economy: An Empirical Analysis“, British Journal of Political Science 39: 449-482.

Hassel, Anke (2003): „The Politics of Social Pacts“, British Journal of Industrial Relations 41: 707-726.

Hassel, A. (2009): „Policies and Politics in Social Pacts in Europe“, European Journal of Industrial Relations, 15: 7-26.

Heinisch, Reinhard (2001): „Defying neoliberal convergence: Austria's successful supply-side corporatism in the 1990s“, Government and Policy 19: 29-44.

Jakob, Johannes (2006): „Die gesetzliche Arbeitslosenversicherung“, in: Ursula Engelen-Kefer (Hg.): Handbuch für Selbstverwalter - Die Arbeit in der Renten-, Kranken-, Pflege-, Unfall-, und Arbeitslosenversicherung. Frankfurt: Bund-Verlag, 21-49.

Jochem, Sven (2009): „Soziale Pakte in Europa“, in: Rehder, Britta, Winter, Thomas \& Willems, Ulrich (Hg.) Interessenvermittlung in Politikfeldern. VS Verlag für Sozialwissenschaften: 210-229. 
Katzenstein, Peter J. (1987): Corporatism and Change. Austria, Switzerland and the Politics of Industry. Ithaca: Cornell University Press.

Klenk, Tanja (2012): „Deutschland: Korporatistische Selbstverwaltung zwischen Staat und Markt“, in: Tanja Klenk; Philine Weyrauch; Alexander Haarmann; Frank Nullmeier (Hg.): Abkehr von Korporatismus? Der Wandel der Sozialversicherungen im europäischen Vergleich. Frankfurt am Main u.a.: Campus, 53-115.

Klenk, Tanja; Weyrauch, Philine; Haarmann, Alexander; Nullmeier, Frank (2012): „Das Ende der korporatistischen Selbstverwaltung?“ in: Tanja Klenk; Philine Weyrauch; Alexander Haarmann; Frank Nullmeier (Hg.): Abkehr von Korporatismus? Der Wandel der Sozialversicherungen im europäischen Vergleich. Frankfurt am Main u.a.: Campus, 1951.

Lehmbruch, Gerhard (1979): „Consociational Democracy, Class Conflict and the New Corporatism“, in: Gerhard Lehmbruch; Philippe C. Schmitter (Hg.): Trends Towards Corporatist Intermediation, London: Sage, 53-61.

Lehmbruch, Gerhard; Schmitter, Philippe C. (Hg.) (1982): Patterns of Corporatist Policy-making. London u.a.: Sage Publications.

Möller, Joachim (2010): „, The German Labor Market Response in the World Recession - Demystifying a Miracle“, Zeitschrift für Arbeitsmarktforschung 42: 325-336.

Obinger, Herbert; Tálos, Emmerich (2006): Sozialstaat Österreich zwischen Kontinuität und Umbau: eine Bilanz der ÖVP/FPÖ/BZÖ-Koalition. Wiesbaden, VS Verlag für Sozialwissenschaften.

Oesch, Daniel (2007): „Weniger Koordination, mehr Markt? Kollektive Arbeitsbeziehungen und Neokorporatismus in der Schweiz seit 1990“, Swiss Political Science Review 13: 337368.

Oesch, Daniel (2011): „Swiss Trade Unions and Industrial Relations after 1990“, in: Christine Trampusch; André Mach (Hg.): Switzerland in Europe. Continuity and Change in the Swiss Political Economy. London: Routledge, 82-102.

Palier, Bruno; Thelen, Kathleen (2010): „Institionalizing Dualism: Complementarities and Change in France and Germany“, Politics and Society 38: 119-148.

Pierson, Paul (2001): „, Introduction: Investigating the Welfare State at Century's End“, in: Paul Pierson (Hg.): The New Politics of the Welfare State. Oxford u.a.: Oxford University Press, 1-16. 
Pizzorno, Alessandro (1978): „Political Exchange and Collective Identity in Industrial Conflict“, in: Colin Crouch; Alessandro Pizzorno (Hg.): The Resurgence of Class Conflict in Western Europe since 1968. London: Macmillan, 277-98.

Pontusson, Jonas, Raess, Damian. (2012) 'How (and Why) Is This Time Different? The Politics of Economic Crisis in Western Europe and the United States', Annual Review of Political Science, 15, 13-33.

Regini, Marino (1984). „The Conditions for Political Exchange: How Concertation Emerged and Collapsed in Britain and Italy“, in: John H. Goldthorpe (Hg.) Order and conflict in contemporary capitalism: studies in the political economy of Western European Nations. Oxford: Clarendon Press, 124-42.

Reisenbichler, Alexander; Morgan, Kimberly J. (2012): „, From “Sick Man” to “Miracle“: Explaining the Robustness of the German Labor Market During and After the Financial Crisis 2008-09”, Politics \& Society 40: 549-579.

Rhodes, Martin (2000): „The Political Economy of Social Pacts: 'Competitive Corporatism' and European Welfare Reform“, in: Gøsta Esping-Andersen; Marino Regini (Hg.): Why Deregulate Labour Markets? Oxford u.a.: Oxford University Press, 165-194.

Rinne, Ulf ; Zimmermann, Klaus F. (2013): „, Is Germany the North Star of Labor Market Policy?“, IZA Discussion Papers.

Schelke, Waltraud (2012): "Policymaking in Hard Times: French and German Responses to the Eurozone Crisis”, in: Nancy Bermeo; Jonas Pontusson (Hg.): Coping with crisis: Government Reactions to the Great Recession. New York: Russell Sage Foundation, ?-?. Schmidt, Manfred G. (2012): Der deutsche Sozialstaat: Geschichte und Gegenwart. München, Beck.

Schmitter, Phillippe; Lehmbruch, Gehrhard (1979): Trends toward Corporatist Intermediation. London u.a.: Sage Publications.

Schmitter, Philippe C. ; Streeck, Wolfgang (1999): „The Organization of Business Interests. Studying the Associative Action of Business in Advanced Industrial Societes“, MPIfG Discussion Paper 1.

Schroeder, Wolfgang (2010): „Geschichte und Funktion der deutschen Arbeitgeberverbände“, in: Wolfgang Schroeder; Bernhard Weßels (Hg.): Handbuch Arbeitgeber- und 
Wirtschaftsverbände in Deutschland.Wiesbaden: VS Verlag für Sozialwissenschaften, 2642.

Schroeder, Wolfgang; Weßels, Bernhard (2010): „Die deutsche Unternehmerverbändelandschaft: vom Zeitalter der Verbände zum Zeitalter der Mitglieder“, in: Wolfgang Schroeder; Bernhard Weßels (Hg.): Handbuch Arbeitgeber- und Wirtschaftsverbände in Deutschland, Wiesbaden: VS Verlag für Sozialwissenschaften, 9-24.

SECO (2012): Bericht des Staatssekretariats für Wirtschaft SECO über die Stabilisierungsmaßnahmen 2009/2010. 15.05.2012. Bern: SECO.

Siaroff, A. (1999): „Corporatism in 24 industrial democracies: Meaning and measurement“, European Journal of Political Research, 36: 175-205.

Silvia, Stephen J. (2010): „Mitgliederentwicklung und Organisationsstärke der Arbeitgeberverbände, Wirtschaftsverbände und Industrieund Handelskammern“, in: Wolfgang Schroeder; Bernhard Weßels (Hg.): Handbuch Arbeitgeber- und Wirtschaftsverbände in Deutschland. Wiesbaden: VS Verlag für Sozialwissenschaften, 169-182.

Streeck, Wolfgang (1991): „On the Institutional Conditions of Diversified Quality Production“, in: Egon Matzner; Wolfgang Streeck (Hg.): Beyond Keynesianism. The SocioEconomics of Production and Employment. London: Edward Elgar, 21-61.

Streeck, Wolfgang; Hassel, Anke (2003): „The Crumbling Pillars of Social Partnership“, West European Politics 26: 101-124.

Streeck, Wolfgang; Hassel, Anke (2003): „The Crumbling Pillars of Social Partnership“, West European Politics 26: 101-124.

Streeck, Wolfgang (2005): „Nach dem Korporatismus: Neue Eliten, neue Konflikte“, MPIfG Working Paper, 05.

Streeck, Wolfgang; Thelen, Kathleen (2005): „Introduction: Institutional Change in Advanced Political Economies“, in: Wolfgang Streeck; Kathleen Thelen (Hg.): Beyond Continuity: Institutional Change in Advanced Political Economies, Oxford u.a.: Oxford University Press, 1-39.

Streeck, Wolfgang; Crouch, Colin (2006): „The Study of Organized Interests: Before ‘The Century’and After“, in: Colin Crouch; Wolfgang Streeck (Hg.): The Diversity of 
Democracy: Corporatism, Social Order and Political Conflict, London: Edward Elgar: 345.

Streeck, Wolfgang (2009): Re-forming Capitalism. Institutional Change in the German Political Economy. Oxford/New York: Oxford University Press.

Tálos, Emmerich; Fink, Marcel (2003): „Sozialpartnerschaft in Österreich: Das korporatistische Modell am Ende?“ in: Sven Jochem; Nico A. Siegel (Hg.): Konzertierung, Verhandlungsdemokratie und Reformpolitik im Wohlfahrtsstaat, Opladen: Leske+Budrich, 194-231.

Trampusch, Christine (2009): Der erschöpfte Sozialstaat. Transformation eines Politikfeldes. Frankfurt am Main: Campus.

Thelen, Kathleen; van Wijnbergen, C. (2003): „The Paradox of Globalization: Labor Relations in Germany and Beyond“, Comparative Political Studies 36: 859-880.

Traxler, Franz (2003): „Bargaining, the State and the Trajectory of Industrial Relations“, European Journal of Industrial Relations 9: 141-161.

Urban, Hans-Peter (2010): „Niedergang oder Comeback der Gewerkschaften - Essay“, APUZ, 13-14, 3-6.

Visser, Jelle (2009): ICTWSS: Database on Institutional Characteristics of Trade Unions, Wage Setting, State Intervention and Social Pacts in 34 countries between1960 and 2007, h3, Version 2. Download unter: http://www.uva-aias.net/208. (Zugriff am 20. 12.2012)

Weishaupt, J. Timo (2010): Germany After a Decade of Social Democrats in Government: The End of the Continental Model? German Politics 19: 105-122.

Weishaupt, J. Timo (2011a): From the Manpower Revolution to the Activation Paradigm. Explaining Institutional Continuity and Change in an Integrating Europe, Amsterdam: Amsterdam University Press.

Weishaupt, J. Timo (2011b): Social Partners and the Governance of Public Employment Services: Trends and Experiences from Western Europe. International Labour Office; Labour Administration and Inspection Programme (LAB/ADMIN) Working Paper 17, Genf: ILO.

Weßels, Bernhard (2000): „Die Entwicklung des deutschen Korporatismus“, Aus Politik und Zeitgeschichte, 7-15. 
Interviews (face-to-face, geführt im Herbst/Winter 2012)

- Deutschland (1): Gesamtmetall (Interview DE-1)

- Österreich (3): Wirtschaftskammer Österreich (Interview AT-1), Kammer für Arbeiter und Angestellte (AT-2), Österreichisches Institut für Wirtschaftsforschung (AT-3)

- Schweiz (3): Arbeitgeberverband (CH-1), Schweizerischer Gewerkschaftsbund (CH-2), Staatssekretariat für Wirtschaft (CH-3) 
Tabelle 1: Wesentliche ökonomische Kennziffern

\begin{tabular}{|c|c|c|c|c|c|c|c|}
\hline & & 2007 & 2008 & 2009 & 2010 & 2011 & 2012 \\
\hline \multirow[t]{3}{*}{ Arbeitslosenquote } & $\overline{\mathrm{DE}}$ & $8,80 \%$ & $7,60 \%$ & $7,90 \%$ & $7,20 \%$ & $6,00 \%$ & $5,60 \%$ \\
\hline & $\mathrm{AT}$ & $4,50 \%$ & $3,90 \%$ & $4,90 \%$ & $4,50 \%$ & $4,20 \%$ & $4,40 \%$ \\
\hline & $\mathrm{CH}$ & $3,70 \%$ & $3,40 \%$ & $4,20 \%$ & $4,70 \%$ & $4,10 \%$ & $4,30 \%$ \\
\hline \multirow{3}{*}{$\begin{array}{l}\text { Veränderung der } \\
\text { Beschäftigung }\end{array}$} & $\mathrm{DE}$ & $2,1 \%$ & $1,4 \%$ & $-0,2 \%$ & $0,7 \%$ & $2,4 \%$ & $0,7 \%$ \\
\hline & AT & $2,1 \%$ & $1,4 \%$ & $-0,4 \%$ & $0,5 \%$ & $1,2 \%$ & $1,0 \%$ \\
\hline & $\mathrm{CH}$ & $1,6 \%$ & $2,4 \%$ & $0,7 \%$ & $0,4 \%$ & $1,8 \%$ & $0,8 \%$ \\
\hline \multirow{3}{*}{$\begin{array}{l}\text { Veränderung des } \\
\text { realen BIP }\end{array}$} & $\overline{\mathrm{DE}}$ & $3,30 \%$ & $1,10 \%$ & $-5,10 \%$ & $4,20 \%$ & $3,00 \%$ & $0,70 \%$ \\
\hline & AT & $3,70 \%$ & $1,40 \%$ & $-3,80 \%$ & $2,10 \%$ & $2,70 \%$ & $0,80 \%$ \\
\hline & $\mathrm{CH}$ & $3,80 \%$ & $2,20 \%$ & $-1,90 \%$ & $3,00 \%$ & $1,90 \%$ & $1,00 \%$ \\
\hline \multirow{3}{*}{$\begin{array}{l}\text { Veränderung der } \\
\text { Reallöhne }\end{array}$} & $\mathrm{DE}$ & $0,10 \%$ & $0,70 \%$ & $-0,40 \%$ & $0,00 \%$ & $1,60 \%$ & $\cdots$ \\
\hline & $\mathrm{AT}$ & $0,70 \%$ & $1,40 \%$ & $1,50 \%$ & $-0,40 \%$ & $-0,30 \%$ & $\ldots$ \\
\hline & $\mathrm{CH}$ & $1,40 \%$ & $0,10 \%$ & $1,80 \%$ & $-0,80 \%$ & $-0,20 \%$ & $\ldots$ \\
\hline \multirow{3}{*}{$\begin{array}{l}\text { Veränderung der } \\
\text { Lohnstückkosten }\end{array}$} & $\mathrm{DE}$ & $-1,53 \%$ & $2,10 \%$ & $6,75 \%$ & $-1,94 \%$ & $1,28 \%$ & $\ldots$ \\
\hline & AT & $0,61 \%$ & $3,08 \%$ & $5,26 \%$ & $-0,60 \%$ & $0,72 \%$ & $\ldots$ \\
\hline & $\mathrm{CH}$ & $1,40 \%$ & $2,75 \%$ & $4,54 \%$ & $-2,32 \%$ & $\ldots$ & $\ldots$ \\
\hline \multirow{3}{*}{$\begin{array}{l}\text { Veränderung der } \\
\text { Produktivität pro } \\
\text { Angestelltem }\end{array}$} & $\mathrm{DE}$ & $2,10 \%$ & $0,00 \%$ & $-5,60 \%$ & $3,90 \%$ & $1,50 \%$ & $\ldots$ \\
\hline & $\mathrm{AT}$ & $1,90 \%$ & $-0,30 \%$ & $-3,40 \%$ & $1,30 \%$ & $1,40 \%$ & $\ldots$ \\
\hline & $\mathrm{CH}$ & $1,20 \%$ & $-0,10 \%$ & $-2,30 \%$ & $2,40 \%$ & $-0,50 \%$ & $\ldots$ \\
\hline
\end{tabular}

Quelle: Eurostat, OECD. 